\title{
Experimental research of structural organization of heat-insulating structural building materials for energy efficient buildings
}

\author{
Said Shaumarov ${ }^{1, *}$, Anvar Adilkhodjaev ${ }^{1, * *}$ and Valeriy Kondrazhenko ${ }^{2,{ }^{* *}}$ \\ ${ }^{1}$ Tashkent Institute of Railway Engineers, Adilkhodjaev st., 1, 100067, Uzbekistan \\ ${ }^{2}$ Russian University of Transport, Minayevskiy Per., 2, 127055, Russia
}

\begin{abstract}
The article presents the results of research to determine the porosity of cellular concrete with different volume mass and strength by the method of "image analysis". A photo-optical method of cellular concrete structure estimation is developed. The dependence of porosity and strength of the samples on the fractal dimension of the cellular concrete structure is obtained.
\end{abstract}

\section{Introduction}

One of the main directions of innovative and energy-saving technologies in the construction industry is the increase of requirements to heat-protective properties of enclosing structures, which can be realized by means of application of developments and effective construction and heat-insulating materials with given properties parameters.

Design of external enclosing structures for energy efficient buildings erected on the territory of the Republic of Uzbekistan from effective construction and thermal insulation materials, as well as research of their structural organization is an urgent task. The greatest effect can be obtained by using inhomogeneous materials consisting of skeleton and cavities or pores filled with air [1-2].

Creation of new construction and thermal insulation materials mainly occurs with the help of empirical methods. Taking into account many factors influencing the peculiarities of structural formation of materials, relying only on experimentally obtained results, the creation of new effective structural and thermal insulation materials is a long and very expensive process. Therefore, the development and development of reliable methods of mathematical modeling to assess the processes occurring in these materials and products to improve the structural and thermal insulation properties of the projected materials is an urgent task [3].

\footnotetext{
* Corresponding author: shoumarovss@gmail.com

${ }^{* *}$ Corresponding author: anvar_1950@mail.ru

${ }^{* * *}$ Corresponding author: valery.kondrashchenko.52@gmail.com
} 


\section{Methods}

In accordance with the methodology for the formation of fractal objects, a numerical experiment was carried out aimed at determining the fractal dimension of the structure of cellular concrete to determine the relationship of the latter with its porous structure. For this, the first step was to build a physical model of cellular concrete with hexagonal, cubic and rhombic packages.

This physical model involves the formation of a spatial framework as a result of successive filling of the volume with pores of a given size [4-5]. At the same time, extremely tight pore laying is achieved due to spontaneous spatial restructuring of the lattice and its compaction with a stepwise increase in the total porosity. Capillary and airentrained pores form a given (hexagonal, cubic or rhombic) interporous material lattice.

The mathematical model of the physical organization of the porous structure of cellular concrete presented above was built on the basis of the prescribed laws of probability density distribution of pores along their section radii for all the above types of packaging so that the porosity of the lattice with the thickness of partitions of $20-60 \mu \mathrm{m}$ varied from $10 \%$ to $90 \%$.

Then, a method based on image analysis of a cellular concrete sample obtained using an «Neophot-21» optical microscope was developed to determine the fractal dimension of the porous surface and further determine the relationship between the fractal dimension and the porosity of cellular concrete.

Image processing was carried out according to a specially developed algorithm, on the basis of which a software package was built, including image input, determining the type of packaging (its proximity to one of three types - hexagonal, cubic or rhombic), searching for boundaries between the pore space and the material (matrix, various types of inclusions, etc.). In addition, one of the main blocks in the program is a block of "quantizing" an image into a given number of levels with the construction for each level of a histogram of the sample's probability density distribution by brightness levels. The last level represents the binary distribution (black - material, white - porosity). To clearly represent the objects subjected to machine image analysis in Fig. 1 shows a micrograph of a fragment of the structure of cellular concrete.

\section{Experimental studies to determine the parameters of cellular concrete structure}

Experimental studies to determine the parameters of the structure (porosity) of cellular concrete were carried out on the basis of the analysis of images from six samples of cellular concrete with known characteristics of its structure. Diagnostics of the parameters of the structure of test samples was carried out by classical methods according to [6-7]. To determine the apparent porosity in the test samples from the existing methods were used: the method of saturation of the sample pores with water during boiling and photo electronic method developed in the Moscow Engineering Construction Institute (MICI) [7].

The apparent porosity for the first method was calculated by the formula $(\%)$ :

$$
P_{\mathrm{\kappa}}=\left[\frac{m_{2}-m_{1}}{m_{2}-m_{3}}\right] \cdot 100,
$$

where $m_{1}$ - mass of sample before saturation with water; $m_{2}$ - mass of sample after saturation with water during air weighing; $\mathrm{m}_{3}$ - mass of sample after saturation during hydrostatic weighing.

Characteristics of porosity of cellular concrete samples were determined by means of photo electronic installation according to MICI method [7].

The surface of the sample was stained with white matte enamel. Then the surface was covered with finely dispersed soot filling all surface irregularities (pores). Then the soot 
was washed away with water. As a result, all the internal surfaces of pores turned out to be painted black, and the surfaces of the pore walls turned out to be white.

Fig. 1 shows images of test samples and histograms of their structure distribution by brightness levels. These histograms are informative enough to describe the mechanism of organization in the forming structure in different types of cellular concrete, which is clearly seen in the figure below.

Table 1 shows the characteristics of samples of different types of cellular concrete (Fig. 1), used as test ones to assess the accuracy of the diagnosis of the structure by the method of analysis of their images, and in Table 2 - the actual quantitative estimates of the accuracy of porosity of cellular concrete on the basis of the developed photo-optical method of image analysis.

Table 1. Characteristics of cellular concrete test samples structure, retrieved by various methods.

\begin{tabular}{|c|c|c|c|c|c|}
\hline \multirow{2}{*}{ № sample } & \multicolumn{3}{|c|}{$\begin{array}{c}\text { According to the manufacturer } \\
\text { data }\end{array}$} & $\begin{array}{c}\text { According to the } \\
\text { MICI methodology }\end{array}$ & $\begin{array}{c}\text { By method of } \\
\text { "image analysis" }\end{array}$ \\
\cline { 2 - 6 } & $\boldsymbol{W , ~ k g / \mathbf { ~ } ^ { \mathbf { 3 } }}$ & $\boldsymbol{R}_{\mathbf{C}, \mathbf{M P a}}$ & $\boldsymbol{P}, \boldsymbol{\%}$ & $\boldsymbol{P ,} \boldsymbol{\%}$ & $\boldsymbol{P}, \boldsymbol{\%}$ \\
\hline 1 & 420 & 2,00 & 80,0 & 79,7 & 81,5 \\
\hline 2 & 500 & 2,31 & 77,8 & 77,2 & 78,4 \\
\hline 3 & 544 & 3,53 & 75,8 & 75,9 & 76,6 \\
\hline 4 & 835 & 7,30 & 65,9 & 66,1 & 67,1 \\
\hline 5 & 840 & 7,38 & 65,3 & 65,2 & 66,2 \\
\hline 6 & 912 & 8,86 & 62,8 & 62,6 & 63,7 \\
\hline
\end{tabular}
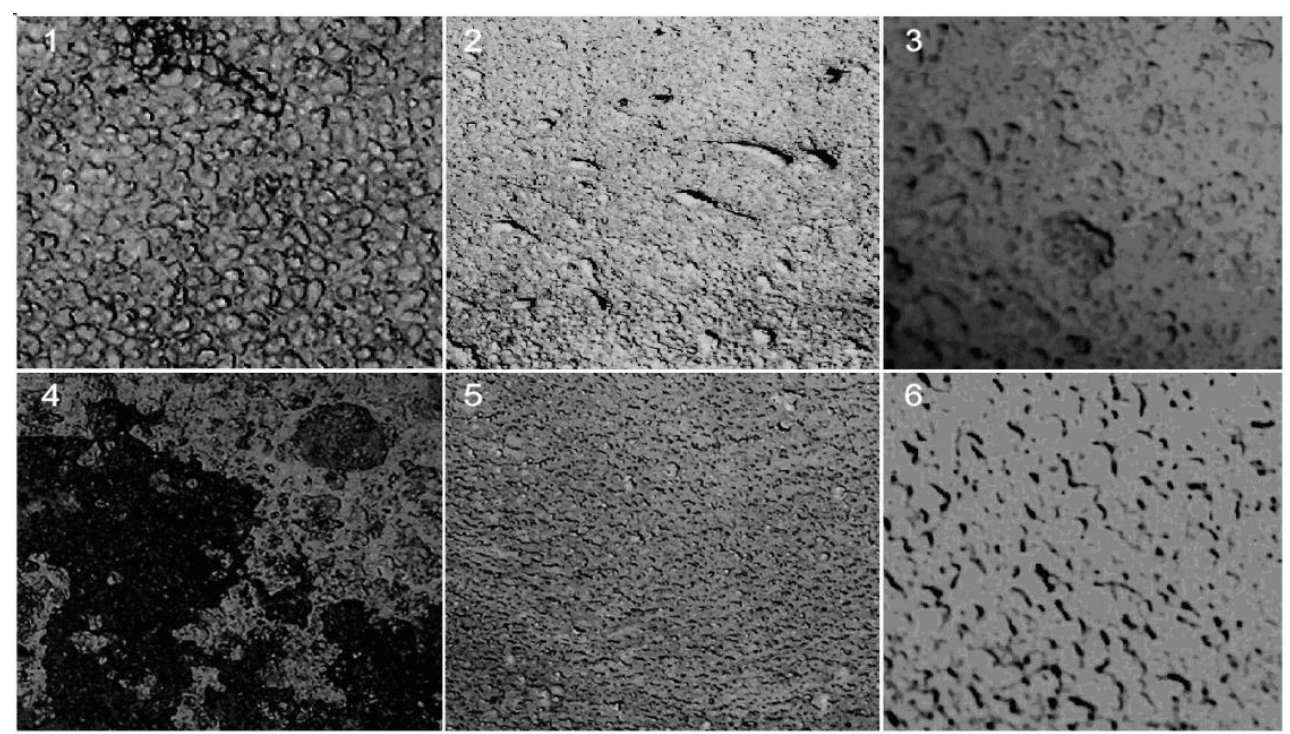

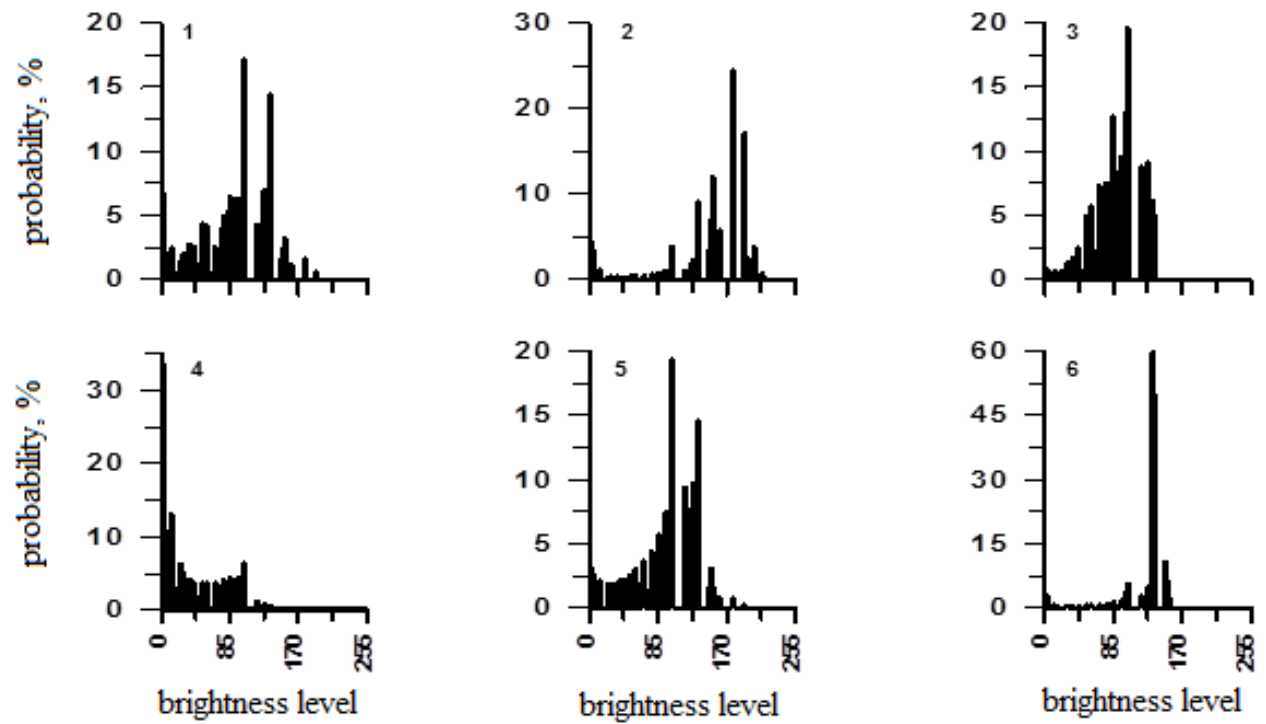

Fig. 1. Test samples of cellular concrete with different volume mass $W$, strength $R_{C}$, and porosity $P$ (table 1) - top panel and their image histograms - bottom panel.

\section{Assessment of the accuracy of cellular concrete porosity determination by "image} analysis" method

As estimates of the accuracy of the method are accepted:

- the difference $\Delta_{i}$ between the value of "truth" of porosity $P_{i}^{R}$, for which the manufacturer's estimation is accepted, and the value of porosity $P_{i}^{\prime}$, obtained by the MICI method and the method of "image analysis".

$$
\Delta_{i}=P_{i}^{R}-P_{i}^{\prime} . . \mathrm{i}=1,2, \ldots, 6 \text { - sample numbers; }
$$

- average error in arithmetic $\bar{\Delta}=\frac{1}{N} \sum_{i=1}^{N} \Delta_{i}$

- absolute maximum error

$$
\begin{aligned}
\left|\Delta_{\max }\right| & =\max \left|\left\{\Delta_{i}\right\}\right| ; \\
\sigma & =\sqrt{\frac{1}{N} \sum_{i=1}^{N}\left(P_{\mathrm{i}}^{\mathrm{R}}-P_{i}^{\prime}\right)^{2}}
\end{aligned}
$$

The estimates in Table 2 show that the control measurements of porosity of cellular concrete samples coincide with the manufacturer's data at the level of random error with a root-mean-square error of 0,288. This is a quite acceptable result that fits into the corresponding [7]. Therefore, this value can be taken as a value of measurement accuracy.

Estimation of the accuracy of porosity of cellular concrete with different volume mass and strength by the method of "image analysis" showed, first of all, the presence of systematic error of the method - the average arithmetic error is different from zero, which indicates a systematic displacement of the calculated porosity in the algorithm of

\begin{tabular}{|c|c|c|c|c|c|c|c|c|}
\hline \multirow{2}{*}{$\begin{array}{c}\text { № } \\
\text { sample }\end{array}$} & \multicolumn{4}{|c|}{ «MICI» methodology } & \multicolumn{4}{|c|}{ «image analysis» methodology } \\
\hline & $\Delta$ & $\Delta_{\max }$ & $\Delta$ & $\sigma$ & $\Delta$ & $\Delta_{\max }$ & $\Delta$ & $\sigma$ \\
\hline
\end{tabular}
processing images of cellular concrete.

Table 2. Assessing the accuracy of cellular concrete porosity by method of "image analysis". 


\begin{tabular}{|l|c|l|l|l|l|l|l|l|}
\hline 1 & 0,150 & & & & $-1,513$ & & & \\
2 & 0,450 & & & & $-0,599$ & & & \\
3 & $-0,250$ & \multirow{2}{*}{0,45} & 0 & 0,288 & $-0,799$ & \multirow{2}{*}{0,513} & $-0,971$ & 0,312 \\
4 & $-0,349$ & & & & $-1,103$ & 1,513 & \\
5 & $-0,051$ & & & & $-0,905$ & & & \\
6 & 0,049 & & & & $-0,912$ & & & \\
\hline
\end{tabular}

At the same time, the mean-square error of the method "image analysis" exceeds the accuracy of measurements, although the value does not differ much from it. The elimination of systematic error in the presence of a representative sample of cellular concrete porosity data is not difficult and is a trivial procedure, although the latter does not change the RMS error. Therefore, an objective indicator of the accuracy of the method is the RMS error, the evaluation of which is quite acceptable from the point of view of the accuracy of the method. Therefore, the developed method for determination of porosity of cellular concretes on the basis of analysis of their image appears, on the one hand, to be quite objective and, on the other hand, incommensurably easier in practical implementation.

The reason for the systematic error of the method, apparently, is related to the resolution of the levels of quantization of the original image and further recognition of the boundaries of the matrix (material) - pores. Further experiments with quantization levels of the initial image were not carried out, since the obtained RMS error $(\sigma=0,312)$ at 8 levels of quantization is acceptable in practical work.

In addition to quantitative estimates of the accuracy of the cellular concrete porosity determination method, the fractal dimensions of the test samples were calculated according to the developed method. The results of the calculation are shown in Table 3.

Table 3. Results of calculation of the fractal dimension D of cellular concrete test samples with different porosity.

\begin{tabular}{|c|c|c|c|c|}
\hline № sample & $\boldsymbol{W}, \mathbf{~ k g} / \mathbf{m}^{\mathbf{3}}$ & $\boldsymbol{R}_{\mathbf{C}}, \mathbf{M P a}$ & $\boldsymbol{P}, \boldsymbol{\%}$ & $\boldsymbol{D}$ \\
\hline 1 & 420 & 2,00 & 80,0 & 1,541 \\
\hline 2 & 500 & 2,31 & 77,8 & 1,620 \\
\hline 3 & 544 & 3,53 & 75,8 & 1,627 \\
\hline 4 & 835 & 7,30 & 65,9 & 1,684 \\
\hline 5 & 840 & 7,38 & 65,3 & 1,685 \\
\hline 6 & 912 & 8,86 & 62,8 & 1,691 \\
\hline
\end{tabular}

\section{Discussion}

Comparison of the obtained results of fractal dimensionality of the structure of real samples of cellular concrete with that of the simulated "random" porosity shows their proximity. Thus, the fractal dimension of cellular concrete with different porosity obtained by the modeling method really corresponds to the fractal dimension of real samples with similar porosity and once again proves the fractal structure of cellular concrete.

In addition, in Fig. 2, the strength surface of cellular concrete is given as a function of fractal dimensionality and porosity. This figure clearly indicates the fact that the fractal dimension of the cellular concrete structure is an informative characteristic of the physical 
properties of the latter, describing all the major parameters: porosity, strength and thermal conductivity.
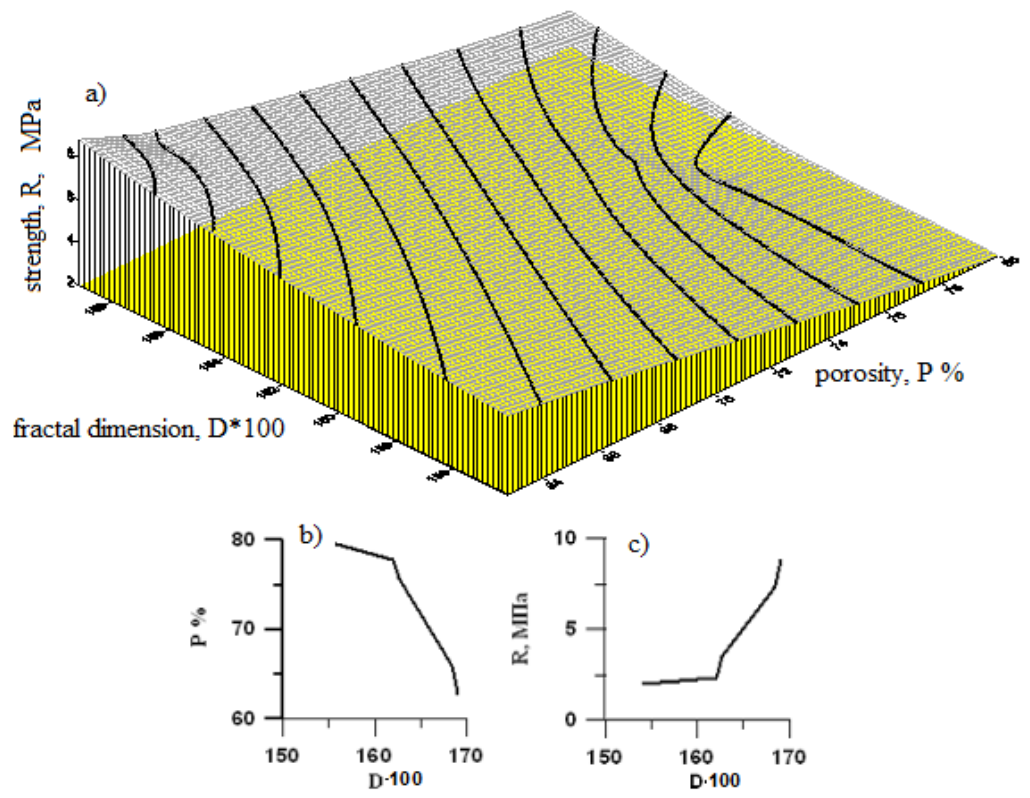

Fig. 2. The surface of cellular concrete strength (a) as a function of fractal dimensionality and porosity; b), c) the dependence of porosity and strength of test samples on the fractal dimension of the cellular concrete structure.

\section{Conclusion}

The nature of the change in the fractal dimension associated with the structure of cellular concrete has the form of an extreme curve - one increasing, the other falling branch, that is, the extreme values of the fractal dimension are placed on the same line with other characteristics. Fractal dimensionality, undoubtedly, is, besides the parameters considered in the article, also a characteristic of the thermal conductivity of cellular concrete.

Thus, the photo-optical method of cellular concrete structure research has been developed on the basis of using the modern approach from the point of view of information technologies. The accuracy of the method is comparable with the accuracy of classical methods, in particular, the diagnosis of porosity of cellular concretes, and its practical implementation is much easier than the latter.

\section{Reference}

1. S.S. Shaumarov, On the issue of increasing energetic efficiency of buildings in railway transport. VIII International Conference "Transport Problems" p. 522-532 (2016)

2. S.S. Shaumarov, Modeling the process of forming the temperature field of the external fencing of buildings on the railway transport. Journal «Scientific and Technical Bulletin of Bryansk State University» 3, 338-346 p. (2018). 
3. A.I. Adilkhodzhaev, S.S. Shaumarov, The issue of improving the energy efficiency of buildings in railway transport. Journal «Modern problems of the transport complex of Russia». Vol. 8, 1. 4-11 pp.(2018).

4. R. Bostanabad, Y. Zhang, X.Li, T. Kearney, C. Brinson, D.W. Apley, W. KamLiu, W. Chen, Computational microstructure characterization and reconstruction: Review of the state-of-the-art techniques. Journal «Progress in Materials Science», Vol. 95, p. 141. (2018).

5. A.I. Adilhodzhayev, S.S. Shaumarov, The issue of thermal renovation of infrastructure of railway transport is evaluated. X International Scientific Conference "Transport Problems" p. 13-18 (2018)

6. A.I. Adilkhodzhaev, I.M. Mahamataliev, S.S. Shaumarov, Theoretical aspects of the structural-imitation modeling of the macrostructure of composite building materials. Journal «Scientific and Technical Bulletin of Bryansk State University» 3, 312-320 p. (2018)

7. Yu. P. Gorlov, Laboratory workshop on the technology of thermal insulation materials. Higher School, 236p. (1992) 